https://helda.helsinki.fi

Does arrival time affect outcomes among severely injured blunt trauma patients at a tertiary trauma centre?

\title{
Brinck, Tuomas
}

2019-11

Brinck , T , Heinänen, M , Söderlund, T, Lefering , R \& Handolin , L 2019 , ' Does arrival time affect outcomes among severely injured blunt trauma patients at a tertiary trauma centre? ' , Injury , vol. 50 , no. 11 , pp. 1929-1933 . https://doi.org/10.1016/j.injury.2019.08.015

http://hdl.handle.net/10138/323893

https://doi.org/10.1016/j.injury.2019.08.015

publishedVersion

Downloaded from Helda, University of Helsinki institutional repository.

This is an electronic reprint of the original article.

This reprint may differ from the original in pagination and typographic detail.

Please cite the original version. 


\title{
Does arrival time affect outcomes among severely injured blunt trauma patients at a tertiary trauma centre?
}

\author{
Tuomas Brinck $^{\mathrm{a}, *}$, Mikko Heinänen ${ }^{\mathrm{a}}$, Tim Söderlund ${ }^{\mathrm{a}}$, Rolf Lefering ${ }^{\mathrm{b}}$, Lauri Handolin ${ }^{\mathrm{a}}$ \\ a Department of Orthopaedics and Traumatology, Trauma Unit, Helsinki University Hospital and University of Helsinki, Finland \\ ${ }^{\mathrm{b}}$ Institute for Research in Operative Medicine (IFOM), University of Witten/Herdecke, Cologne, Germany
}

\section{A R T I C L E I N F O}

\section{Article history:}

Accepted 10 August 2019

\section{Keywords:}

Severe injuries

Blunt trauma

Outcome

Admission time

Quality control

\begin{abstract}
A B S T R A C T
Background and aims: We aimed to determine whether the outcome of severely injured patients differs based on admission time (office hours vs. non-office hours) at a tertiary trauma centre without an inhouse trauma surgeon consultant available at all times. We also studied subgroups of patients presenting with a New Injury Severity Score (NISS) $\geq 25$ and patients experiencing major bleeding.

Patients and methods: This trauma registry study consisted of severely injured patients (NISS > 15) with blunt trauma treated between 2006 and 2017 at a single institute. Causes of deaths were obtained from autopsy reports and classified as resulting from brain injury; exsanguination; multi-organ failure, adult respiratory distress syndrome, or sepsis; or other.

Results: Among 1853 patients, 497 (27\%) were admitted during office hours (OH) and 1356 (73\%) during non-office hours (NOH). Further subgroup analysis consisted of $211 \mathrm{OH}$ and $611 \mathrm{NOH}$ patients with NISS $\geq 25$, and $51 \mathrm{OH}$ and $154 \mathrm{NOH}$ patients experiencing major bleeding. The 30-day in-hospital mortality was 3.8\%-7.4\% lower in the NOH groups. We found no significant differences between the study groups in neither the standardised mortality ratio (SMR, defined as the ratio of observed to expected mortality) nor in the causes of death. In both groups, the primary cause of death resulted from brain injury.

Conclusions: We found that arrival time did not affect mortality among patients with severe blunt trauma treated at a tertiary trauma centre without an in-house trauma surgeon consultant available at all times. Thus, this type of unit can maintain a standard of care during non-office hours by investing in precise treatment protocols and continuous education. However, our results do not apply to penetrating trauma injury patients.
\end{abstract}

(c) 2019 Elsevier Ltd. All rights reserved.

\section{Introduction}

Trauma occurs unexpectedly, often at night and at the weekend. Severe trauma patients arriving at hospital warrant rapid diagnostics, the marked utilisation of resources including both personnel and infrastructure, and effective coordination and decision-making. Given that the number and experience of personnel in the emergency, radiology, and interventional radiology departments as well as operating theatres are usually highest during standard office hours, the question arises whether a severely injured patient arriving at hospital during out-of-office

\footnotetext{
* Corresponding author at: Department of Orthopaedics and Traumatology, Helsinki University Hospital, Topeliuksenkatu 5, PB 266, FI-00029 HUS, Helsinki, Finland.

E-mail address: tuomas.brinck@hus.fi (T. Brinck).
}

hours has a worse prognosis compared with those arriving when resources are at their peak.

Previous research on the effect of arrival time on trauma patient outcomes varied. For instance, some studies noted 'after-hours' arrivals increased mortality or complications [1-4], whilst others showed no difference in the outcomes between patients arriving during or outside business hours [5-10].

Previously at our institute, traumatic deaths in the emergency room (ER) were retrospectively reviewed. During a nine-year study period (1998-2006), seven potentially preventable deaths were identified and attributed to failures in the surgical decisionmaking process, resulting in futile non-operative treatment or a delay in surgical bleeding control. Five of the potentially preventable deaths occurred at night, four of which were likely due to a lack of experience. These findings highlighted the importance of staffing an experienced senior trauma surgeon at the hospital at all times [11]. However, ten years after that study, our major tertiary trauma centre continues to lack an in-house 
consultant trauma surgeon during non-office hours, and acute decision-making often falls to on-call residents only.

Therefore, we aimed to determine whether the outcomes among severely injured patients differed based on the admission time (office hours vs. non-office hours) at our institute. We hypothesised that patients arriving during non-office hours (NOH) experienced worse outcomes; we further hypothesised that this difference would be more pronounced in subgroups of very severely injured patients and patients experiencing major bleeding.

\section{Patients and methods}

The Helsinki Trauma Registry (HTR) provided data on severely injured patients [New Injury Severity Score (NISS) $>15$ ] treated from 2006 through 2017. HTR is a local trauma registry in the trauma unit of the Helsinki University Hospital, which centralises the treatment of severe blunt injuries among adult patients $(\geq 16$ years) with a catchment area of 1.8 million inhabitants (25\% of the total Finnish population) extending for $200 \mathrm{~km}$ in southern Finland.

In our study, we excluded patients with a penetrating injury or unknown injury mechanism, isolated burns, referred patients, and patients with an isolated head injury [defined as a head Abbreviated Injury Scale (AIS) $\geq 3$, no other AIS $\geq 2$ ]. Patients included in this study were further analysed in two subgroups: patients with very severe injuries (defined as NISS $\geq 25$ ) and patients experiencing major bleeding. The latter subgroup consisted of patients who received $>2$ red blood cell units in the emergency department and presented with a shock index (pulse rate divided by systolic blood pressure) at admission of $\geq 0.9$ and/or a systolic blood pressure at admission of $\leq 90$.

In each subgroup, patients were divided into two groups depending upon the admission time: patients admitted during office hours $(\mathrm{OH}, 7: 00-15: 30$ on working days) and patients admitted during non-office hours ( $\mathrm{NOH}, 15: 31-6: 59$ on weekdays, all patients admitted at the weekends or on Finnish bank holidays).

We compared the following parameters between the $\mathrm{OH}$ and NOH groups: age, sex, injury mechanism, ISS, NISS, injury pattern (head, thorax, abdomen, and extremities), blood transfusion, and cause of death.

The cause of death was obtained from autopsy reports. Finnish law requires an autopsy be performed on all individuals dying due to an accident, dying under obscure circumstances, and dying or suspected to have died due to violence (both self-inflicted and otherwise). The autopsy report was available for each deceased patient. Deaths were classified as due to brain injury (CNS); exsanguination; multi-organ failure (MOF), adult respiratory distress syndrome (ARDS), or sepsis; or other. A death due to CNS was defined as a brain injury and/or cervical cord injury incompatible with life regardless of other injuries. Exsanguination was determined as the cause of death if major bleeding occurred regardless of any possible attempts to control it, and when CNS was not applicable. In cases of uncertainty or if two equal causes of deaths were listed on the autopsy report (e.g., CNS and bleeding), patient charts were reviewed by one author (TB) and an estimation of the clinically more relevant cause of death was determined and selected as the sole cause of death for the purposes of analysis.

We defined the outcome as 30-day in-hospital mortality (death during the first hospital admission within 30 days of the initial trauma) and early mortality (within $12 \mathrm{~h}$ of the injury). Expected mortality was calculated using the average value of the individual prognoses derived from the Revised Injury Severity Classification score II (RISC II; [12]).

All comparisons were calculated using only the information available, whereby no imputations were made. We used the means and standard deviations (SDs) for all descriptive analyses for all continuous variables, and percentages for categorical variables. The standardised mortality ratio (SMR) was defined as the ratio of the observed 30-day in-hospital mortality to the expected mortality, deriving the 95\% confidence interval (CI) using the respective interval for the observed mortality.

We used SPSS (version 23, IBM Inc., Armonk NY, USA) for all comparisons and statistical analyses. The administrative board of the Helsinki University Hospital approved the study protocol.

\section{Results}

Amongst the sample of 1853 patients, 497 (27\%) patients were admitted during office hours $(\mathrm{OH})$ and 1356 (73\%) patients during non-office hours (NOH). Further subgroup analysis found that 51 $\mathrm{OH}$ and $154 \mathrm{NOH}$ patients presented with major bleeding. The subgroup analysis of very severely injured (NISS $\geq 25$ ) patients consisted of $211 \mathrm{OH}$ and $611 \mathrm{NOH}$ patients, respectively. Some patients were included in both subgroups (that is, patients experiencing major bleeding and patients very severely injured). Fig. 1 summarises the inclusion and exclusion criteria applied to the study sample.

In each subgroup, the majority of patients were male $(66 \%-$ $75 \%$ ). The difference in ISS was less than 2 between the $\mathrm{OH}$ and

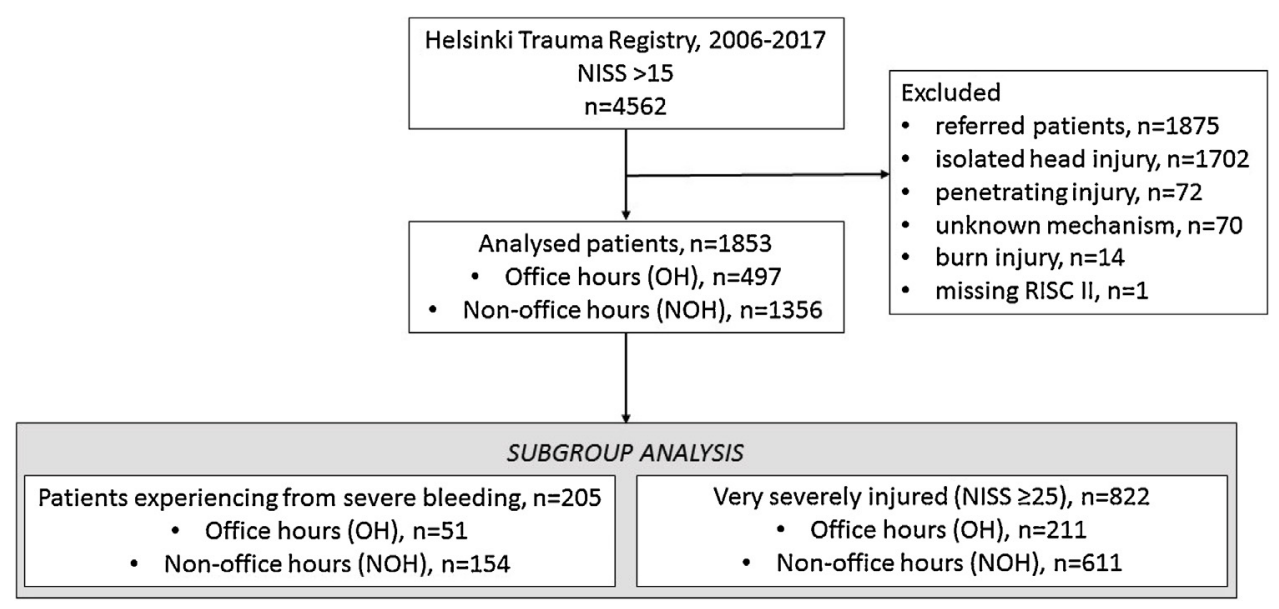

Fig. 1. Flowchart of the inclusion and exclusion criteria for patients.

Abbreviations: NISS, New Injury Severity Score; RISC II, Revised Injury Severity Classification Score, version II. 
NOH groups, whilst this difference was less than 3 regarding NISS. The mean age of $\mathrm{NOH}$ patients was $7.0-10.9$ years younger in each group. In the $\mathrm{NOH}$ groups, $8 \%-19 \%$ more patients were injured in a motorbike accident, whilst $6 \%-10 \%$ fewer patients sustained pedestrian injuries (Table 1 ).

The 30-day in-hospital mortality was 3.8\%-7.4\% lower in the $\mathrm{NOH}$ groups. In addition, we found no significant difference in SMR (defined as the ratio of observed to expected mortality) between the study groups, indicated by the overlapping 95\% CI (Table 2).

The distribution of the causes of deaths between the $\mathrm{OH}$ and NOH groups did not differ. In both groups, the major cause of death was CNS (brain injury), responsible for $67 \%(\mathrm{OH})$ and $63 \%(\mathrm{NOH})$ of all deaths (Table 3 ).

\section{Discussion}

In our study on severely injured patients treated at a single tertiary trauma centre, we sought to determine whether blunt trauma patients arriving during non-office hours (NOH) had a worse prognosis compared with those arriving during office hours $(\mathrm{OH})$. We found no differences in the adjusted mortality between study groups, including subgroup comparisons between patients presenting with major bleeding and patients presenting with very severe injuries (NISS $\geq 25$ ).

We found that three out of four major trauma patients arrived during non-office hours. Patients who arrived during NOH were younger (41 vs. 48 years), perhaps partially explained by highenergy leisure time activities more common amongst younger people, thereby exposing them to severe injuries. In addition, based on our previous experience, many younger major trauma patients in particular arrive under the influence of alcohol and/or drugs, typical at night and at the weekend. Yet, severe work-related injuries likely concentrate during office hours, although we did not analyse this here. Aside from younger patients and more motorbike injuries during $\mathrm{NOH}$ and more pedestrian injuries concentrated during office hours, no other notable differences existed between the study groups. Thus, we considered the study groups comparable to one another.

Contrary to our original hypothesis, we found no difference in the adjusted outcomes between the study groups. We found this rather surprising for several reasons. In our institute, the emergency department on-call surgeon is defined as the trauma team leader, who is almost always a resident. Residents are doctors with a minimum of three years of general surgical training. At the beginning of residency, the residents need to successfully pass a simulation, where they act as a trauma team leader treating a severely injured patient. The emphasis of this simulation is on institute's protocols and structured communication. Our trauma care protocols are based on European Trauma Course (ETC) Guidelines. In addition to the trauma team leader, multidisciplinary trauma team involves nine persons: an anesthesiologist, a radiologist, three nurses, and additional personnel from radiology department and laboratory. Neurosurgeon is involved in case of an intracranial injury. No emergency medicine specialists exist in our institute.

During office hours, the trauma team leader is supervised and guided by an experienced surgeon consultant familiar with acute decision-making and treatment options for major trauma patients. However, during off-hours the trauma team leader is often responsible for first-line decision-making since no in-house consultant surgeon is available at all times. Whilst present in the hospital, the on-call consultant surgeon is often in the operating theatre and, thus, unavailable to the emergency department. Furthermore, the consultant surgeon might be specialised in spinal, upper- or lower-limb surgery and possibly not as experienced in the acute decision-making process for a patient with, for instance, a bleeding organ injury. Our trauma centre does not have a hybrid theatre and, thus, the trauma team leader must take time-sensitive decisions, such as whether a bleeding patient is treated in an operating theatre by means of damage control surgery or in an angiography suite with embolisation. In addition, we do not have an interventional radiology team present at the hospital around the clock. When necessary, the team arrives from home, potentially causing an unnecessary delay during non-office hours.

Little et al.'s [13] recent study from a major trauma centre in London found no significant difference in the 30-day mortality when directly comparing weekday and weekend admittances, yet noted a significantly higher mortality among patients admitted on Friday and Saturday compared to the remainder of the week. Since their unit features consistent trauma team staffing levels and resources throughout the week, as well as an around-the-clock ED consultant, the increased mortality appeared likely explained by more severe head trauma injuries. In our study isolated head injuries were excluded. Amongst those patients included, although we did not analyse Fridays and Saturdays separately, we found no

Table 1

Patient characteristics.

\begin{tabular}{|c|c|c|c|c|c|c|}
\hline & \multicolumn{2}{|l|}{ All Patients } & \multicolumn{2}{|l|}{ Bleeders } & \multicolumn{2}{|c|}{ Very Severe Injuries } \\
\hline & Office hours & Non-office hours & Office hours & Non-office hours & Office hours & Non-office hours \\
\hline Number of cases & 497 (27\%) & $1356(73 \%)$ & $51(25 \%)$ & $154(75 \%)$ & $211(26 \%)$ & $611(74 \%)$ \\
\hline Age (mean, SD), in years & $48.4(20.5)$ & $41.3(19.0)$ & $48.0(22.2)$ & $37.1(18.0)$ & $46.3(21.7)$ & $39.3(19.3)$ \\
\hline Male sex (\%) & $72 \%$ & $74 \%$ & $67 \%$ & $66 \%$ & $72 \%$ & $75 \%$ \\
\hline \multicolumn{7}{|l|}{ Mechanism: } \\
\hline car & $23 \%$ & $24 \%$ & $18 \%$ & $26 \%$ & $21 \%$ & $23 \%$ \\
\hline motorbike & $10 \%$ & $19 \%$ & $2 \%$ & $21 \%$ & $8 \%$ & $16 \%$ \\
\hline bicycle & $9 \%$ & $7 \%$ & $4 \%$ & $3 \%$ & $7 \%$ & $5 \%$ \\
\hline pedestrian & $12 \%$ & $6 \%$ & $16 \%$ & $6 \%$ & $14 \%$ & $6 \%$ \\
\hline high fall & $30 \%$ & $29 \%$ & $39 \%$ & $37 \%$ & $32 \%$ & $23 \%$ \\
\hline low fall & $3 \%$ & $5 \%$ & $0 \%$ & $3 \%$ & $4 \%$ & $5 \%$ \\
\hline other & $13 \%$ & $10 \%$ & $21 \%$ & $48 \%$ & $18 \%$ & $22 \%$ \\
\hline ISS (mean, SD) & $26.1(12.2)$ & $25.9(11.2)$ & $37.5(14.1)$ & $37.5(13.6)$ & $36.9(11.3)$ & $35.5(9.8)$ \\
\hline New ISS (mean, SD) & $31.4(13.2)$ & $31.7(12.7)$ & $44.0(14.8)$ & $43.7(14.1)$ & $42.5(12.6)$ & $42.0(11.3)$ \\
\hline Head injury (AIS 3+) & $40 \%$ & $44 \%$ & $37 \%$ & $43 \%$ & $58 \%$ & $59 \%$ \\
\hline Thorax trauma (AIS $3+$ ) & $69 \%$ & $63 \%$ & $75 \%$ & $79 \%$ & $80 \%$ & $76 \%$ \\
\hline Abdominal trauma (AIS 3+) & $23 \%$ & $21 \%$ & $47 \%$ & $51 \%$ & $35 \%$ & $34 \%$ \\
\hline Extremities (AIS 3+) & $36 \%$ & $34 \%$ & $80 \%$ & $60 \%$ & $51 \%$ & $41 \%$ \\
\hline Blood transfusion (until ICU) & $23 \%$ & $21 \%$ & $100 \%$ & $100 \%$ & $38 \%$ & $35 \%$ \\
\hline
\end{tabular}

Abbreviations: SD, standard deviation; ISS, Injury Severity Score; AIS, Abbreviated Injury Scale; ICU, intensive care unit. 
Table 2

Patient outcomes.

\begin{tabular}{|c|c|c|c|c|c|c|}
\hline & \multicolumn{2}{|l|}{ All Patients } & \multicolumn{2}{|l|}{ Bleeders } & \multicolumn{2}{|c|}{ Very Severe Injuries } \\
\hline & Office hours & Non-office hours & Office hours & Non-office hours & Office hours & Non-office hours \\
\hline Number of cases & 497 & 1356 & 51 & 154 & 211 & 611 \\
\hline Early deaths $(12 \mathrm{~h})$ & $4.2 \%$ & $4.0 \%$ & $19.6 \%$ & $17.5 \%$ & $9.5 \%$ & $8.2 \%$ \\
\hline Died in-hospital (30 days) & $11.9 \%$ & $8.1 \%$ & $31.4 \%$ & $24.0 \%$ & $23.7 \%$ & $16.5 \%$ \\
\hline RISC II prognosis (\%) & $10.1 \%$ & $8.7 \%$ & $31.1 \%$ & $27.3 \%$ & $19.1 \%$ & $15.8 \%$ \\
\hline SMR $(95 \% \mathrm{CI})$ & $1.18(0.89-1.46)$ & $0.93(0.77-1.10)$ & $1.01(0.60-1.42)$ & $0.88(0.63-1.13)$ & $1.24(0.94-1.54)$ & $1.05(0.86-1.23)$ \\
\hline
\end{tabular}

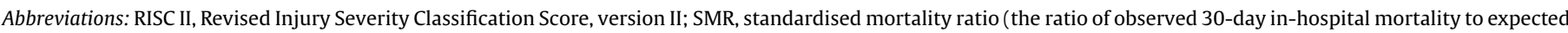
mortality); CI, confidence interval.

Table 3

Causes of deaths amongst all deceased patients based on autopsy reports and medical records.

\begin{tabular}{lll}
\hline & Office hours $(\mathrm{OH})$ & Non-office hours (NOH) \\
\hline Deceased patients, \% (n) & $12(59)$ & $8(110)$ \\
CNS, \% (n) & $67(39)$ & $63(71)$ \\
Bleeding, \% (n) & $16(10)$ & $17(19)$ \\
MOF, sepsis, or ARDS, \% (n) & $7(4)$ & $8(9)$ \\
Other, \% (n) & $11(6)$ & $11(11)$ \\
\hline
\end{tabular}

Abbreviations: CNS, central nervous system; MOF, multi-organ failure; ARDS, adult respiratory distress syndrome.

significant difference in either the severity of brain injuries (as indicated by the similar proportion of AIS $3+$ head injuries) or autopsy-verified causes of deaths between the $\mathrm{OH}$ and $\mathrm{NOH}$ groups. Brain injury represented the major cause of death in both groups, responsible for $67 \%(\mathrm{OH})$ and $63 \%(\mathrm{NOH})$ of deaths.

Our study agrees with finding previously noted by Carr et al. [8], who found that following guidelines and protocols related to organising a trauma system, whereby trauma centres must be fully functional regardless of the time of day, likely explains the encouraging outcomes amongst trauma patients during off-hours. Furthermore, implementing regular trauma team simulations, morbidity and mortality meetings, and practical damage control surgery training amongst residents at our institute, as well as providing international courses such as the European Trauma Course $^{\mathrm{TM}}$ (ETC) and the Definitive Surgical Trauma Care ${ }^{\mathrm{TM}}$ (DSTC) course, all aid in training surgeons to take quick decisions and act appropriately in emergency situations.

Furthermore, our findings related to similar outcomes amongst the $\mathrm{OH}$ group agree with the majority of previous studies. For instance, installing an around-the-clock in-house attending supervising surgeon at an academic level-I trauma centre had no effect on the death rate of trauma patients [14]. However, this practice significantly reduced the spike of preventable complications previously observed at the beginning of the academic year when new interns and fellows arrive at a teaching hospital [14]. A study conducted at another level-I trauma centre investigated the outcome of severely injured patients admitted at night or at the weekend before and after the implementation of an on-call inhouse attending. Whilst no benefit of an in-house attending was observed on overall mortality, the incidence of preventable deaths and serious adverse events decreased [15].

We must consider several weaknesses to this study. Here, we did not record adverse events, such as futile operative treatments, unnecessary delays in investigations and procedures, or postoperative complications. In addition, the study protocol limited us to a comparison of the use or availability of resources during office and non-office hours, which possibly played a major role in prompt acute care. We also acknowledge other limitations related to registry studies, such as the retrospective design. We must also note that our study encompassed only blunt trauma patients, and our findings do not necessarily extend to major penetrating torso injuries. The strengths of our study, however, include its focus on only one tertiary trauma centre, the availability of all autopsy records, and the previously validated, high-quality data characteristic of the Helsinki Trauma Registry [16].

\section{Conclusions}

To conclude, we found that arrival time does not affect mortality amongst patients with severe blunt trauma treated at a tertiary trauma centre without an in-house trauma surgeon consultant available at all times. Units without an in-house trauma consultant can maintain a standard of care during non-office hours by investing in precise treatment protocols and continuous education. Our results, however, do not apply to penetrating trauma patients.

\section{Declaration of Competing Interest}

The authors declare no potential conflicts of interest with respect to the research, authorship, and/or publication of this article.

\section{References}

[1] Egol KA, Tolisano AM, Spratt KF, Koval KJ. Mortality rates following trauma: the difference is night and day. J Emerg Trauma Shock 2011;4(2):178-83.

[2] Mitra B, Cameron PA, Fitzgerald MCB, Bernard S, Moloney J, Varma D, et al. "After-hours" staffing of trauma centres and outcomes among patients presenting with acute traumatic coagulopathy. MJA 2014;201:588-91, doi: http://dx.doi.org/10.5694/mja13.00235.

[3] Barbosa Lde S, dos Reis Junior GS, Chaves RZ, Solla DJ, Canedo LF, Cunha AG. Night admission is an independent risk factor for mortality in trauma patients-a systemic error approach. Rev Col Bras Cir 2015;42(4):209-14.

[4] Di Bartolomeo S, Marino M, Ventura C, Trombetti S, De Palma R. A population based study on the night-time effect in trauma care. Emerg Med J 2014;31:808-12, doi:http://dx.doi.org/10.1136/emermed-2013-202338.

[5] Busse JW, Bhandari M, Devereaux PJ. The impact of time of admission on major complications and mortality in patients undergoing emergency trauma surgery. Acta Orthop Scand 2004;75(3):333-8.

[6] Guly HR, Leighton G, Woodford M, Bouamra O, Lecky F. The effect of working hours on outcome from major trauma. Emerg Med J 2006;23:276-80, doi: http://dx.doi.org/10.1136/emj.2005.028746.

[7] Laupland KB, Ball CG, Kirkpatrick AW. Hospital mortality among major trauma victims admitted on weekends and evenings: a cohort study. J Trauma Manag Outcomes 2009;3:8, doi:http://dx.doi.org/10.1186/1752-2897-3-8.

[8] Carr BG, Jenkins P, Branas CC, Wiebe DJ, Kim P, Schwab CW, et al. Does the trauma system protect against the weekend effect? J Trauma 2010;69:1042-8, doi:http://dx.doi.org/10.1097/TA.0b013e3181f6f958.

[9] Ono Y, Ishida T, Iwasaki Y, Kawakami Y, Inokuchi R, Tase C, et al. The off-hour effect on trauma patients requiring subspecialty intervention at a community hospital in Japan: a retrospective cohort study. Scand J Trauma Resusc Emerg Med 2015:23:20, doi:http://dx.doi.org/10.1186/s13049-015-0095-1.

[10] Pape-Köhler CIA, Simanski C, Nienaber U, Lefering R. External factors and the incidence of severe trauma: time, date, season and moon. Injury 2014;45:939, doi:http://dx.doi.org/10.1016/j.injury.2014.08.027.

[11] Söderlund T, Tulikoura I, Niemelä M, Handolin L. Traumatic deaths in the emergency room: a retrospective analysis of 115 consecutive cases. Eur J Trauma Emerg Surg 2009;35:455, doi:http://dx.doi.org/10.1007/s00068-009 8179-0. 
[12] Lefering R, Huber-Wagner S, Nienaber U, Maegele M, Bouillon B. Update of the trauma risk adjustment model of the TraumaRegister DGU ${ }^{\mathrm{TM}}$ : the Revised Injury Severity Classification, version II. Crit Care 2014;18(5):476.

[13] Little Z, Bethel J, Clements J, Trompeter AJ. Major trauma: does weekend attendance increase 30-day mortality? Injury 2019;50:351-7, doi:http://dx. doi.org/10.1016/j.injury.2018.12.007.

[14] Inaba K, Hauch A, Branco BC, Cohn S, Teixeira PG, Recinos G, et al. The impact of in-house attending surgeon supervision on the rates of preventable complications and death at the start of the new academic year. Am Surg 2013;79:1134-9.

[15] Durham R, Shapiro D, Flint L. In-house trauma attendings: is there a difference? Am J Surg 2005;190:984-90.

[16] Heinänen M, Brinck T, Handolin L, Mattila VM, Söderlund T. Accuracy and coverage of diagnosis and procedural coding in the Finnish Hospital Discharge Register: comparison to patient files and the Helsinki Trauma Registry. Scand J Surg 2017;106:269-77, doi:http://dx.doi.org/10.1177/1457496916685236. 